\title{
Experimental demonstration and proposal of silicon-on-insulator octagonal microresontor devices
}

\author{
Chao Li and Andrew W. Poon
}

Department of Electrical and Electronic Engineering, The Hong Kong University of Science and Technology, Clear Water Bay, Hong Kong SAR, China Tel: (852)-2358-7905, fax: (852)-2358-1485, email: eeawpoon@ust.hk

\begin{abstract}
We demonstrate the first lateral waveguide-coupled octagonal microresonator filters in silicon-on-insulator (SOI). We also propose a SOI electro-optical modulator by use of a round-cornered octagonal microresonator with a selectively integrated ring-shaped metal-oxide-semiconductor capacitor.

OCIS codes: (230.5750) Resonators; (230.3990) Microstructure devices
\end{abstract}

Silicon-on-insulator (SOI) lateral waveguide-coupled microresonators in the shape of circular microring [1] and racetrack microring [2] have been attracting considerable interest for channel add-drop, optical switch, and modulator applications. However, the short interaction length between the high-index contrast microring curved sidewall and straight waveguide sidewall imposes a technological challenging submicron $(\sim 0.1 \mu \mathrm{m}-\sim 0.2 \mu \mathrm{m})$ air-gap separation for the lateral coupling. Although racetrack shaped microring with flat sidewalls was proposed as an alternative microresonator design, the transitions between the racetrack straight waveguide and curved waveguide sections still pose significant cavity loss. Previously, we proposed and demonstrated polygonal microresonators, including octagonal microresonators [3], in high-index contrast silicon nitride-on-silica substrate as alternative microresonator designs. Such polygonal microresonators have the key advantage of flat cavity sidewalls for the ease of lateral coupling, yet without the complications of curved cavity sidewalls. Most recently, we also demonstrated that silicon nitride octagonal microresonators with rounded corners have increased resonance $Q$ factors in comparison with the sharp-cornered design. [3].

(a)

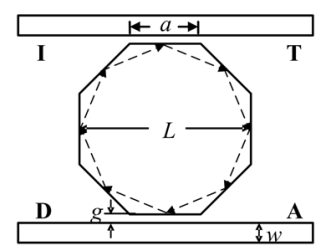

(b)

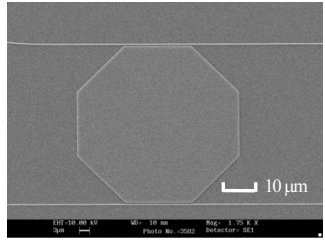

(c)

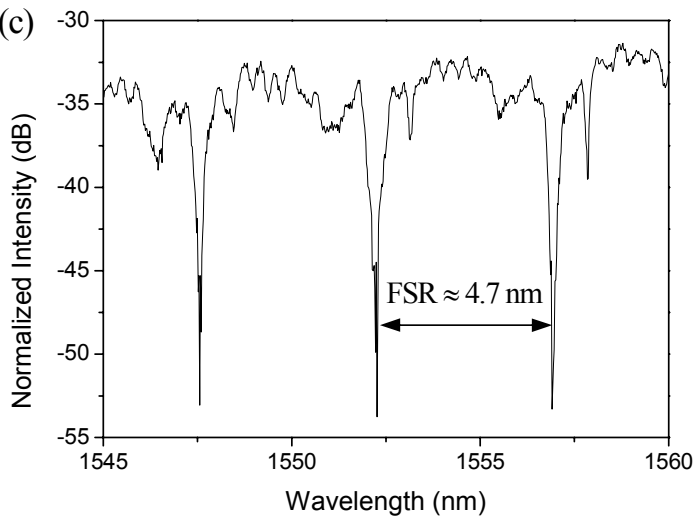

Fig. 1. (a) Schematic of the lateral waveguide-coupled octagonal microresonator channel add-drop filter. Dashed arrows illustrate an 8-bounce ray orbit. (b) Scanning electron micrograph of the fabricated filter in SOI. $\mathrm{L}=50 \mu \mathrm{m}, \mathrm{a}=20.71 \mu \mathrm{m}, \mathrm{w}=0.35 \mu \mathrm{m}$ and $\mathrm{g}=$ $0.35 \mu \mathrm{m}$. (c) Measured TE-polarized throughput-port spectrum.

In this summary, we report our initial demonstration of lateral waveguide-coupled octagonal microresonator filters in SOI. We study experimentally and numerically such octagonal microresonator filters with sharp-cornered and round-cornered cavity designs. We also propose a compact SOI electro-optical modulator design by use of a round-cornered octagonal microresonator with a selectively integrated ring-shaped metal-oxide-semiconductor (MOS) capacitor.

Figure 1 (a) shows the schematic of the lateral waveguide-coupled octagonal microresonator channel add-drop filter. The dashed arrows depict an 8-bounce ray orbit that can be wavefront-matched and can result in resonances. Such resonance orbits can be input and output-coupled along the entire flat sidewalls.

Figure 1 (b) shows the top-view scanning electron micrograph (SEM) of the fabricated filter. We employed standard 
silicon microelectronic fabrication processes. The filter layout was defined by i-line photolithography, and transferred onto a $0.2-\mu \mathrm{m}$ thick SOI device layer by $\mathrm{CHF}_{3}$-based plasma etching. The SOI BOX layer is $3 \mu \mathrm{m}$. The etched depth was $\sim 0.15$ $\mu \mathrm{m}$. The sidewall-to-sidewall distance $L$ is $\sim 50 \mu \mathrm{m}$, the waveguide width $w$ is $\sim 0.35 \mu \mathrm{m}$, and the waveguide-to-resonator air-gap separation $g$ is $\sim 0.35 \mu \mathrm{m}$. The sidewall interaction length $a$ is $\sim 20.71 \mu \mathrm{m}$.

Figure 1 (c) shows the initially measured TE-polarized ( $\vec{E} / /$ the chip) throughput-port spectrum. We demonstrate nearly singlemode resonances that have the maximum $Q$ value $\approx 4000$ and coupling efficiency $\approx 99 \%$. The measured free spectral range (FSR) is $\approx 4.7 \mathrm{~nm}$, which is consistent with the calculated FSR of an 8-bounce ray-orbit mode in a silicon refractive index of 3.5 [3]. The finesse is about 12 .

(a)

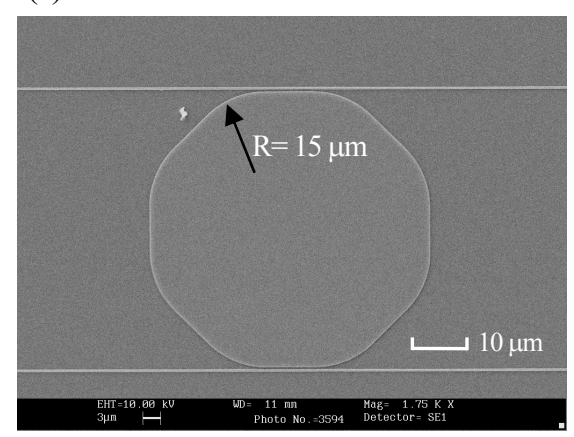

(b)

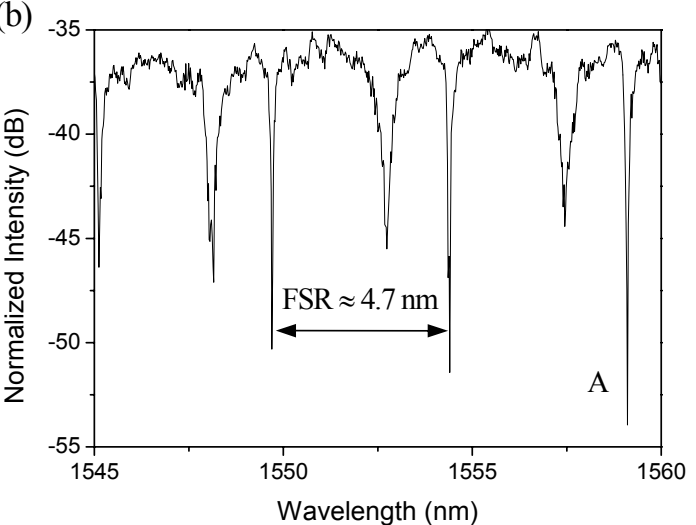

Fig. 2. (a) Scanning electron micrograph of a SOI lateral waveguide-coupled round-cornered octagonal microresonator channel add-drop filter. $\mathrm{L}=50 \mu \mathrm{m}, \mathrm{R}=15 \mu \mathrm{m}, \mathrm{a}=8.3 \mu \mathrm{m}, \mathrm{w}=0.35 \mu \mathrm{m}$ and $\mathrm{g}=0.35 \mu \mathrm{m}$. (b) Measured TE-polarized throughput spectrum.

We study the effects of rounded cavity corners. Figure 2(a) shows the top-view SEM of the fabricated SOI lateral waveguide-coupled octagonal microresonator filters with rounded corners. An arc of $45^{\circ}$ with a radius $R \approx 15 \mu \mathrm{m}$ is applied to each corner. The round-cornered design also reduces the sidewall interaction length $a$ to $\sim 8.3 \mu \mathrm{m}$.

Figure 2(b) shows the initially measured TE-polarized multimode (bi-mode) throughput-port spectrum of the round-cornered microresonator filter. In contrast with the sharp-cornered octagonal microresonator spectrum (Fig. 1 (c)), the sharp mode A has an enhanced Q of $\approx 10000$, a slightly compromised coupling efficiency of $\approx 97 \%$, and an improved finesse of 31. The FSR remains $\approx 4.7 \mathrm{~nm}$.

(a)

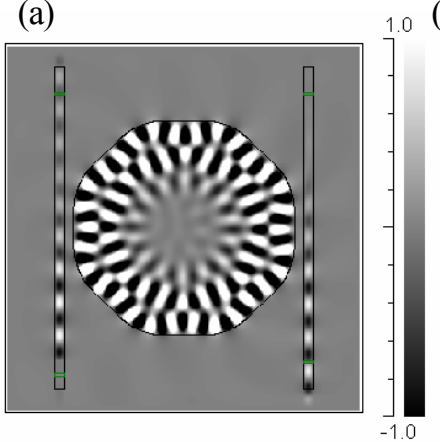

(b)

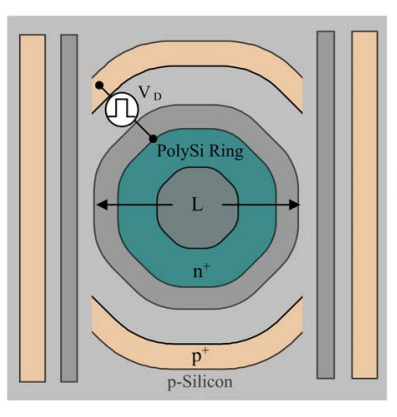

(c)

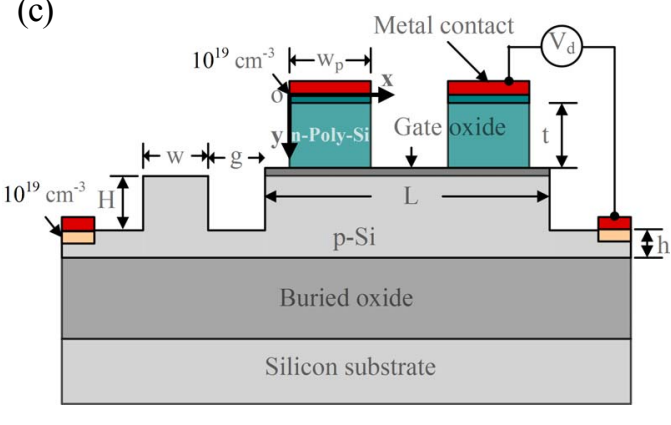

Fig. 3. (a) FDTD simulated TE-polarized mode-field pattern of a lateral waveguide-coupled round-cornered octagonal microresonator filter. The mode field suggests a high-order whispering-gallery like mode. (b) Schematic of the proposed SOI electro-optical modulator by use of a lateral waveguide-coupled round-corner octagonal microresonator with a selectively integrated ring-shaped MOS capacitor. (c) Schematic cross -sectional view of the MOS-capacitor integrated octagonal microdisk.

In order to help visualize the mode-field patterns of octagonal microresonator devices, we performed two-dimensional finite-difference time-domain (FDTD) simulations of a small-sized device. Figure 3(a) shows a representative simulated 
TE-polarized mode-field pattern of a lateral waveguide-coupled round-cornered octagonal microresonator add-drop filter. The cavity shape and waveguide-width follows the measured device (Fig. 2(a)), yet with an $L=5 \mu \mathrm{m}$. We chose a refractive index-contrast of 3.5 in the simulations. The mode-field pattern resembles a traveling-wave high-order whispering-gallery (WG) mode.

Here we propose a SOI electro-optical microresonator-based modulator by use of a lateral waveguide-coupled round-cornered octagonal microresonator with a selectively integrated ring-shaped vertical MOS capacitor [4]. Such design should make possible a monolithically integrated SOI microresonator-based high-speed modulator, yet with potentially less stringent fabrication constraint along the coupling region should we employ conventional circular microring or microdisk resonators.

Figures 3 (b) and 3(c) illustrate the schematic of the top-view and cross-sectional view of the modulator concept. We can selectively integrate a ring-shaped MOS capacitor along the microresonator rim region in order to spatially overlap the accumulated free carriers at the gate oxide with the WG-like mode field (Fig. 3). The resonance wavelengths, Q and extinction ratios can then be tuned by use of free carrier plasma dispersion effect. Our MOS capacitor design comprises an n-doped polysilicon top layer, a $\sim 10$-nm thin gate oxide layer, and a $\mathrm{p}$-doped silicon bottom layer. The $\mathrm{p}^{+}$regions for electrical contact can be in the slab layer outside the microresonator in order to minimize optical absorption loss. The n-type polysilicon ring width can be sufficiently wide to enable the accumulated carriers to spatially overlap with the mode field and for the practical concern of opening contact holes. The device is cladded by a low-temperature oxide (LTO) layer for electrical isolation.

In summary, we experimentally demonstrated the first octagonal microresonator-based devices in SOI. Our initial measurements showed that a lateral waveguide-coupled $50-\mu \mathrm{m}$ sized round-cornered octagonal microresonator filter has reasonable filter characteristics - a Q of about $10^{4}$, a coupling efficiency exceeding $97 \%$, and a finesse of about 31 . We also outline a design of a SOI electro-optical modulator based on round-corned octagonal microresonator with a selectively integrated ring-shaped MOS capacitor. Further experimental work on the passive octagonal microresonator filters and design and fabrication of the proposed MOS-integrated microresonator modulators are in progress.

\section{References}

[1] J. Niehusmann, A. Vörckel, P. H. Bolivar, T. Wahlbrink, W. Henschel, H. Kurz, "Ultrahigh-quality-factor silicon-on-insulator microring resonator", Opt Lett, Vol. 29, pp. 2861-2863, 2004

[2] P. Dumon, W. Bogaerts, V. Wiaux, J. Wouters, S. Beckx, J. Van Campenhout, D. Taillaert, B. Luyssaert, P. Bienstman, D. Van Thourhout, and R. Baets, "Low-loss SOI photonic wires and ring resonators fabricated with deep UV lithography," IEEE Photon. Technol. Lett., vol. 16, no. 5, pp. 1328-1330, 2004.

[3] C. Li, N. Ma, and A. W. Poon, "Experimental demonstration of waveguide-coupled round-cornered octagonal microresonators in silicon nitride," Opt. Lett., vol. 30,pp. 546-548, 2005.

[4] A. Liu, R. Jones, L. Liao, D. Samara-Rubio, D. Rubin, O. Cohen, R. Nicolaescu and M. Paniccia, “A high-speed silicon optical modulator based on a metal-oxide-semiconductor capacitor", Nature, vol. 427, pp. 615-618, 2004. 